difference method was used to solve the flow equations. The exact solution to the thermal transport problem of different types of nanofluids was given by Turkyilmazoglu [6]. Sandeep and Gnaneswara [7] scrutinized the effects of nonlinear thermal radiation on the flow of a $\mathrm{Cu}$-water nanofluid. The Runge-Kutta Newton-Raphson algorithm was used to solve the flow equations. Dhlamini et al. [8] discussed the second-grade nanofluid flow over a nonlinearly stretching sheet. Das et al. [9] solved the equations for the Casson nanofluid flow and heat transfer in a porous medium using a spectral quasi-linearization approximation. El-Aziz [10] studied the effect of variable viscosity on the flow and heat transfer of a power-law nanofluid. Recently, Das et al. [11] examined the influence of variable fluid properties on nanofluid flow over a wedge with surface slip.

The studies above considered the viscosity and thermal conductivity of the nanofluid to be a function of nanoparticle size. However, these properties may also change with temperature (Ogunseye et al. [12]). To accurately predict the heat transfer properties of nanofluids, it is important to consider a viscosity and thermal conductivity model that depends on the nanoparticle size and temperature. In recent years, scientists have proposed numerous viscosity and thermal conductivity models that are nanoparticle size and temperature dependent, and among these, the studies by Masoud et al. [13] and Hassani et al. [14] are worth mentioning.

Lie group symmetry analysis is a powerful technique for finding similarity solutions to a given set of partial differential equations. Using Lie group analysis, we can find similarity transformations that reduces $m$ independent variables of a partial differential equation into $m-1$ independent variables. Many authors have applied Lie group symmetry analysis to fluid flow models. Akgül and Pakdemirli [15] studied the transient flow of a power-law fluid using the Lie group symmetry analysis. Jalil and Asghar [16] analysed the boundary layer flow of a Powell-Eyring fluid using the Lie group symmetry analysis. The scaling group of transformations, a special form of the Lie group symmetry, was used by Rehman et al. [17] in studying the heat and mass transfer in a Powell-Eyring fluid flow past a stretching plate. Afify and El-Aziz [10] discussed the scaling group for the flow and heat transfer behaviour in a powerlaw nanofluid. Other studies using the Lie group analysis are reported in [18-21].

The main focus of this study is the flow and heat transfer analysis in a non-Newtonian nanofluid with variable viscosity and thermal conductivity using the Lie group symmetry analysis. The Powell-Eyring model [22] is adopted due to its diverge advantages over other non-Newtonian fluid models. The model is derived from the molecular theory of fluids and not on empirical relations. Further, under low and high shear rates the Powell-Eyring fluid is reduced to a Newtonian fluid. A considerable number of studies on the Powell-Eyring fluid flow with constant viscosity have been reported by several authors such as Javed et al. [23], Jalil and Asghar [16], Hayat et al. [24], Mahanthesh et al. [25], Agbaje et al. [26] and Ramzan et al. [27]. However, the Powell-Eyring nanofluid flow with variable properties is yet to be considered. The second-order partial differential equation that models the thermal transportation problem is transformed into an ordinary differential equation using the classical Lie group symmetry approach. The equations are solved using an efficient iterative spectral local linearization method. The viscosity and thermal conductivity adopted here are derived from experimental data. The findings in this study may be useful for engineers in the design of heat exchangers and thermal solar collectors.

\section{Formulation of the problem}

A steady, two-dimensional, laminar flow of an incompressible Powell-Eyring nanofluid past a stretching surface is considered. The flow is restricted to the region $\bar{y}>0$, and the stretching velocity is assumed to be $u_{w}(\bar{x})$. The nanofluid is aluminium oxide $\mathrm{Al}_{2} \mathrm{O}_{3}$-water system. Further, the physical properties of the fluid are assumed to vary with the nanoparticle size and temperature.

Following the work of Javed et al. [23] and under the usual boundary layer approximation, the continuity, equations of momentum and energy balance for the Powell-Eyring nanofluid are as follows,

$\frac{\partial \bar{u}}{\partial \bar{x}}+\frac{\partial \bar{v}}{\partial \bar{y}}=0$,

$$
\begin{aligned}
\rho_{n f}\left(\bar{u} \frac{\partial \bar{u}}{\partial \bar{x}}+\bar{v} \frac{\partial \bar{u}}{\partial \bar{y}}\right)= & \frac{\partial}{\partial \bar{y}}\left(\mu_{n f} \frac{\partial \bar{u}}{\partial \bar{y}}\right)+\frac{1}{\beta \gamma} \frac{\partial^{2} \bar{u}}{\partial \bar{y}^{2}} \\
& -\frac{1}{2 \beta \gamma^{3}}\left(\frac{\partial \bar{u}}{\partial \bar{y}}\right)^{2} \frac{\partial^{2} \bar{u}}{\partial \bar{y}^{2}}
\end{aligned}
$$

$$
\begin{aligned}
\rho_{n f} C_{n f}\left(\bar{u} \frac{\partial \bar{T}}{\partial \bar{x}}+\bar{v} \frac{\partial \bar{T}}{\partial \bar{y}}\right)= & \frac{\partial}{\partial \bar{y}}\left(k_{n f} \frac{\partial \bar{T}}{\partial \bar{y}}\right) \\
& +\frac{16 \sigma_{s}}{3 k_{m}} \frac{\partial}{\partial \bar{y}}\left(\bar{T}^{3} \frac{\partial \bar{T}}{\partial \bar{y}}\right) .
\end{aligned}
$$

The relevant boundary conditions to Eqs. (1)-(3) are

$$
\begin{aligned}
\bar{y}=0: & \bar{u}=U_{0} u_{w}\left(\frac{\bar{x}}{L}\right), \quad \bar{v}=V_{0} v_{w}\left(\frac{\bar{x}}{L}\right), \quad \bar{T}=T_{w^{\prime}} \\
\bar{y} \rightarrow \infty: & \bar{u} \rightarrow 0, \quad \bar{T} \rightarrow T_{\infty},
\end{aligned}
$$

\section{SN Applied Sciences}


where $(\bar{u}, \bar{v})$ are the velocity components in the $(\bar{x}, \bar{y})$ directions, $\beta$ and $\gamma$ are Powell-Eyring fluid material constants, $\bar{T}$ is the nanofluid temperature, $\sigma_{s}$ is the Stefan-Boltzmann constant, $k_{m}$ is the mean absorption coefficient, $U_{0}$ and $V_{0}$ are the reference velocities, $L$ is the characteristic length, $T_{w}$ is the wall temperature and $T_{\infty}$ is the nanofluid temperature far away from the wall.

The density $\left(\rho_{n b}\right)$ and specific heat capacity $\left(C_{n b}\right)$ of the nanofluid are given by the expressions (see Khanafer and Vafai [28]),

$$
\begin{aligned}
& \rho_{n f}=(1-\phi) \rho_{b f}+\phi \rho_{p^{\prime}} \\
& C_{n f}=(1-\phi) \rho_{b f} C_{b f}+\phi \rho_{p} C_{p^{\prime}}
\end{aligned}
$$

where the subscripts $b f$ and $p$ represent the base fluid and nanoparticle, respectively, and $\phi$ is the volume fraction of the nanoparticle.

Following Masoud et al. [13], the nanofluid viscosity is defined by

$$
\begin{aligned}
& \frac{\mu_{n f}}{\mu_{b f}}=\exp \left[m+\alpha\left(\frac{\bar{T}}{T_{\infty}}\right)\right. \\
& \left.\quad+\beta_{1} \phi\left(1+\frac{2 r}{d_{p}}\right)^{3}+\gamma_{1}\left(\frac{\mathrm{d}_{p}}{1+r}\right)\right] .
\end{aligned}
$$

The thermal conductivity is determined by the new empirical correlations proposed by Hassani et al. [14], which is expressed as,

$$
\begin{aligned}
\frac{k_{n f}}{k_{b f}}= & \frac{k_{p}+2 k_{b f}-2\left(k_{b f}-k_{p}\right) \phi}{k_{p}+2 k_{b f}+\left(k_{b f}-k_{p}\right) \phi}+5 \\
& \times 10^{4} \frac{\beta_{2} \phi \rho_{b f} C_{b f}}{k_{b f}} \sqrt{\frac{\kappa \bar{T}}{\rho_{p} d_{p}}} f(\bar{T}, \phi),
\end{aligned}
$$

where

$$
\begin{aligned}
f(\bar{T}, \phi)= & \left(2.8217 \times 10^{-2} \phi+3.917 \times 10^{-3}\right)\left(\frac{\bar{T}}{T_{\infty}}\right) \\
& -\left(3.0669 \times 10^{-2} \phi+3.91123 \times 10^{-3}\right),
\end{aligned}
$$

where $\mu$ is the viscosity, $k$ is the thermal conductivity, $m$ is a factor that depends on the nanoparticles, the base fluid and their interaction, $\alpha, \beta_{1}, \beta_{2}$ and $\gamma_{1}$ are empirical parameters determined from experimental data, $d_{p}$ is the diameter of the nanoparticle, $r$ is the capping layer thickness and $\kappa$ is the Boltzmann constant. The values of these empirical parameters are given in Table 1, and the thermo-physical properties of water and aluminium oxide $\mathrm{Al}_{2} \mathrm{O}_{3}$ are presented in Table 2.
Table 1 Empirical parameters for aluminium oxide $\mathrm{Al}_{2} \mathrm{O}_{3}$-water nanofluid $[13,14]$

\begin{tabular}{llllll}
\hline$m$ & $\alpha$ & $\beta_{1}$ & $\gamma_{1}$ & $\beta_{2}$ & Volume fraction \\
\hline 0.72 & -0.485 & 14.94 & 0.0105 & $8.4407(100 \phi)^{-1.07304}$ & $1 \% \leq \phi \leq 10 \%$
\end{tabular}

Table 2 Thermo-physical properties of water and aluminium oxide $\mathrm{Al}_{2} \mathrm{O}_{3}$

\begin{tabular}{llll}
\hline & $\rho$ & $c_{p}\left(\mathrm{~J} \mathrm{~kg}^{-1} \mathrm{~K}\right)$ & $k\left(\mathrm{Wm}^{-1} \mathrm{~K}\right)$ \\
\hline Water fluid & 997.1 & 4179 & 0.613 \\
$\mathrm{Al}_{2} \mathrm{O}_{3}$ & 3970 & 765 & 40 \\
\hline
\end{tabular}

Equations (1)-(4) are nondimensionalized by introducing the following dimensionless variables:

$x=\frac{\bar{x}}{L}, \quad y=\frac{\bar{y}}{L}\left(\frac{U_{0} \rho_{b f} L}{\mu_{b f}}\right)^{\frac{1}{2}}, \quad u=\frac{\bar{u}}{U_{0}}$,

$v=\frac{\bar{v}}{U_{0}}\left(\frac{U_{0} \rho_{b f} L}{\mu_{b f}}\right)^{\frac{1}{2}}, \quad T=\frac{\bar{T}-T_{\infty}}{T_{w}-T_{\infty}}$.

We define the stream function $\psi$ as

$u=\frac{\partial \psi}{\partial y}, \quad v=-\frac{\partial \psi}{\partial x}$.

Substituting Eqs. (8) and (9) into Eqs. (2)-(4) yields:

$$
\begin{gathered}
\frac{\partial}{\partial y}\left(\exp \left(A_{1}+\alpha[1+\Delta T]\right) \frac{\partial^{2} \psi}{\partial y^{2}}\right)+\lambda \frac{\partial^{3} \psi}{\partial y^{3}}-\lambda \delta\left(\frac{\partial^{2} \psi}{\partial y^{2}}\right)^{2} \frac{\partial^{3} \psi}{\partial y^{3}} \\
-A_{2}\left(\frac{\partial \psi}{\partial y} \frac{\partial^{2} \psi}{\partial \partial x \partial y}-\frac{\partial \psi}{\partial x} \frac{\partial^{2} \psi}{\partial y^{2}}\right)=0
\end{gathered}
$$

$\frac{\partial}{\partial y}\left(\left[A_{3}+A_{4}[1+\Delta T]^{\frac{3}{2}}-A_{5}[1+\Delta T]^{\frac{1}{2}}\right] \frac{\partial T}{\partial y}\right)$

$$
+\operatorname{Nr} \frac{\partial}{\partial y}\left([1+\Delta T]^{3} \frac{\partial T}{\partial y}\right)-\operatorname{Pr}_{6}\left(\frac{\partial \psi}{\partial y} \frac{\partial T}{\partial x}-\frac{\partial \psi}{\partial x} \frac{\partial T}{\partial y}\right)=0
$$

$y=0: \quad \frac{\partial \psi}{\partial y}=u_{w}(x), \quad \frac{\partial \psi}{\partial x}=F_{w} v_{w}(x), \quad T=1$,

$y \rightarrow \infty: \quad \frac{\partial \psi}{\partial y} \rightarrow 0, \quad T \rightarrow 0$.

where $\lambda$ and $\delta$ are fluid parameters, Pr is the Prandtl number, $\mathrm{Nr}$ is the thermal radiation parameter, $\Delta$ is the temperature difference, ${ }_{F} w$ is the suction or injection parameter and $A_{i},(i=1, \ldots, 5)$ are constants. These parameters and constants are defined as 


$$
\begin{aligned}
& A_{1}=m+\beta_{1} \phi\left(1+\frac{2 r}{d_{p}}\right)^{3}+\gamma_{1}\left(\frac{d_{p}}{1+r}\right), \\
& A_{2}=\left((1-\phi)+\frac{\rho_{p}}{\rho_{b f}} \phi\right), \quad A_{3}=\frac{k_{p}+2 k_{b f}-2\left(k_{b f}-k_{p}\right) \phi}{k_{p}+2 k_{b f}+\left(k_{b f}-k_{p}\right) \phi}, \\
& F_{w}=-V_{0}\left(\frac{L \rho_{f}}{U_{0} \mu_{\infty}}\right) \\
& A_{4}=\left(1.4109 \times 10^{3} \phi^{2}+1.9585 \times 10^{2} \phi\right) \frac{\beta_{2} \rho_{b f} C_{b f}}{k_{b f}} \sqrt{\frac{\kappa T_{\infty}}{\rho_{p} d_{p}}}, \\
& A_{5}=\left(1.5334 \times 10^{3} \phi^{2}+1.9556 \times 10^{2} \phi\right) \frac{\beta_{2} \rho_{b f} C_{b f}}{k_{b f}} \sqrt{\frac{\kappa T_{\infty}}{\rho_{p} d_{p}}}, \\
& A_{6}=(1-\phi)+\frac{\rho_{p} C_{p}}{\rho_{b f} C_{b f}} \phi, \quad \lambda=\frac{1}{\mu_{b f} \beta \gamma}, \quad \delta=\frac{U_{0}^{3} \rho_{b f}}{2 L C^{2} \mu_{b f}}, \\
& \operatorname{Pr}=\frac{C_{b f} \mu_{b f}}{k_{b f}}, \quad \mathrm{Nr}=\frac{16 \sigma_{s} T_{\infty}^{3}}{3 k_{m} k_{b f}}, \quad \Delta=\theta_{w}-1, \quad \theta_{w}=\frac{T_{w}}{T_{\infty}} .
\end{aligned}
$$

\section{Lie symmetry analysis}

In this section, we seek the similarity solution to Eqs. (10)-(12) using the Lie symmetry group approach. Finding symmetry group is equivalent to finding the infinitesimal generator that renders Eqs. (10)-(12) invariant. We consider a one-parameter group Lie group of infinitesimal transformations with Lie group parameter $\varpi$ defined as:

$$
\begin{aligned}
x^{*} & =x+\varpi \xi_{1}(s, y, \psi, T)+\mathcal{O}\left(\varpi^{2}\right), \\
y^{*} & =y+\varpi \xi_{2}(s, y, \psi, T)+\mathcal{O}\left(\varpi^{2}\right), \\
\psi^{*} & =\psi+\varpi \varphi_{1}(s, y, \psi, T)+\mathcal{O}\left(\varpi^{2}\right), \\
T^{*} & =T+\varpi \varphi_{2}(s, y, \psi, T)+\mathcal{O}\left(\varpi^{2}\right),
\end{aligned}
$$

the infinitesimal generator is prolongated to first, second and third derivatives, and it is defined by:

$$
\begin{aligned}
X= & \xi_{1} \frac{\partial}{\partial x}+\xi_{2} \frac{\partial}{\partial y}+\varphi_{1} \frac{\partial}{\partial \psi}+\varphi_{2} \frac{\partial}{\partial T}+\varphi_{1 x} \frac{\partial}{\partial \psi_{x}}+\varphi_{1 y} \frac{\partial}{\partial \psi_{y}} \\
& +\varphi_{1 x y} \frac{\partial}{\partial \psi_{x y}}+\varphi_{1 y y} \frac{\partial}{\partial \psi_{y y}}+\varphi_{1 y y y} \frac{\partial}{\partial \psi_{y y y}} \\
& +\varphi_{2 x} \frac{\partial}{\partial T_{x}}+\varphi_{2 y} \frac{\partial}{\partial T_{y}}+\varphi_{2 y y} \frac{\partial}{\partial T_{y y}}
\end{aligned}
$$

where

$$
\begin{aligned}
\varphi_{1 R}= & D_{R} \varphi_{1}-\psi_{x} D_{R} \xi_{1}-\psi_{y} D_{R} \xi_{2 \prime} \\
\varphi_{2 R}= & D_{R} \varphi_{2}-\varphi_{2 x} D_{R} \xi_{1}-\varphi_{2 y} D_{R} \xi_{2 \prime} \\
\varphi_{1 S R}= & D_{R} \varphi_{1 S}-\psi_{S x} D_{R} \xi_{1}-\psi_{S y} D_{R} \xi_{2 \prime} \\
\varphi_{2 S R}= & D_{R} \varphi_{2 S}-\psi_{S x} D_{R} \xi_{1}-\psi_{S y} D_{R} \xi_{2 \prime} \\
D_{x}= & \frac{\partial}{\partial x}+\psi_{x} \frac{\partial}{\partial \psi}+T_{x} \frac{\partial}{\partial x}+\psi_{x x} \frac{\partial}{\partial \psi_{x}} \\
& +T_{x x} \frac{\partial}{\partial T_{x}}+\psi_{x y} \frac{\partial}{\partial \psi_{y}}+\ldots, \\
D_{y}= & \frac{\partial}{\partial y}+\psi_{y} \frac{\partial}{\partial \psi}+T_{y} \frac{\partial}{\partial y}+\psi_{y y} \frac{\partial}{\partial \psi_{y}} \\
& +T_{y y} \frac{\partial}{\partial T_{y}}+\psi_{x y} \frac{\partial}{\partial \psi_{x}}+\ldots,
\end{aligned}
$$

$R, S$ represent either $x$ or $y$ depending on the component.

Using $X$ on Eqs. (10) and (11), we obtain the following infinitesimals after some algebraic simplification:

$\xi_{1}=c_{1} x+c_{2}, \quad \xi_{2}=\frac{1}{3} c_{1} y+g(x), \quad \varphi_{1}=\frac{2}{3} c_{1} \psi+c_{3}, \quad \varphi_{2}=0$.

where $c_{j}(j=1, \ldots, 3)$ are arbitrary constants. Thus, we obtain a three-dimensional space of operator. By setting $g(x)=0$ and any of two the constants to zero, the following infinitesimal generators can be obtained:

$x_{1}=x \frac{\partial}{\partial x}+\frac{y}{3} \frac{\partial}{\partial y}+\frac{2}{3} \frac{\partial}{\partial \psi}, \quad x_{2}=\frac{\partial}{\partial x}, \quad x_{3}=\frac{\partial}{\partial \psi}$.

The implication of Eq. (18) is that Eqs. (10) and (11) admit three one-parameter transformation groups. $X_{1}$ corresponds to scaling group of transformation, while $X_{2}$ and $X_{3}$ are translation groups of transformation.

Applying $X$ to the boundary conditions, Eq. (12) yields:

$\begin{aligned} \frac{\mathrm{d} u_{w}}{\mathrm{~d} x} & =\frac{c_{1}}{3\left(c_{1} x+c_{2}\right)} u_{w} \\ \frac{\mathrm{d} v_{w}}{\mathrm{~d} x} & =-\frac{c_{2}}{3\left(c_{1} x+c_{2}\right)} v_{w}\end{aligned}$

which implies that:

$u_{w}(x)=k_{1}\left(c_{1} x+c_{2}\right)^{\frac{1}{3}}$,
$v_{w}(x)=k_{2}\left(c_{1} x+c_{2}\right)^{-\frac{1}{3}}$,

where $k_{1}$ and $k_{2}$ are constants of integration. 
Obviously, $X_{2}$ and $X_{3}$ do not have an invariant solution; hence, we consider only $X_{3}$. Under $X_{1}$, Eq. (20) is transformed into:

$u_{x}(x)=x^{\frac{1}{3}}$ and $v_{w}(x)=x^{-\frac{1}{3}}$.

The characteristic equation to $X_{1}$ is:

$\frac{\mathrm{d} x}{x}=\frac{3 \mathrm{~d} y}{y}=\frac{3 \mathrm{~d} \psi}{2 \psi}=\frac{\mathrm{d} T}{0}$,

on solving Eq. (22) , we obtain the following similarity variables and function:

$\eta=y x^{-\frac{1}{3}}, \quad \psi=x^{\frac{2}{3}} f(\eta), \quad T=\theta(\eta)$.

Finally, substituting Eqs. (21) and (22) into Eqs. (10) and (12) yields:

$$
\begin{aligned}
& \left(\exp \left(A_{1}+\alpha(1+\Delta \theta)\right)+\lambda\right) f^{\prime \prime \prime}+\alpha \Delta \exp \left(A_{1}+\alpha(1+\Delta \theta)\right) \theta^{\prime} f^{\prime \prime} \\
& -\lambda \delta\left(f^{\prime \prime}\right)^{2} f^{\prime \prime \prime}+A_{2}\left(\frac{2}{3} f f^{\prime \prime}-\frac{1}{3} f^{\prime 2}\right)=0
\end{aligned}
$$$$
\left(A_{3}+A_{4}(1+\Delta \theta)^{\frac{3}{2}}-A_{5}(1+\Delta \theta)^{\frac{1}{2}}+\operatorname{Nr}(1+\Delta \theta)^{3}\right) \theta^{\prime \prime}
$$$$
+\Delta\left(\frac{3 A_{4}}{2}(1+\Delta \theta)^{\frac{1}{2}}-\frac{A_{5}}{2}(1+\Delta \theta)^{-\frac{1}{2}}+3 \operatorname{Nr}(1+\Delta \theta)^{2}\right) \theta^{\prime 2}
$$$$
+\frac{2}{3} \operatorname{Pr} A_{6} f \theta^{\prime}=0
$$

$\eta=0: \quad f^{\prime}=1, \quad f=F_{w^{\prime}} \quad \theta=1$,

$\eta \rightarrow \infty: \quad f^{\prime} \rightarrow 0, \quad \theta \rightarrow 0$.

Pantokratoras [29] pointed out that assuming a uniform Prandtl number when thermo-physical properties are temperature dependent may lead to unrealistic results. Hence, to take care of this paradox, the Prandtl number of the nanofluid is defined as

$$
\begin{aligned}
\operatorname{Pr}_{v}= & \frac{\mu_{n f} C_{n f}}{k_{n f}}=\frac{A_{6} \exp \left(A_{1}+\alpha(1+\Delta \theta)\right)}{A_{3}+A_{4}(1+\Delta \theta)^{\frac{3}{2}}-A_{5}(1+\Delta \theta)^{\frac{1}{2}}} \\
& \frac{C_{b f} \mu_{b f}}{k_{b f}} \\
= & \frac{\mu_{n f} C_{n f}}{k_{n f}}=\frac{A_{6} \exp \left(A_{1}+\alpha(1+\Delta \theta)\right)}{A_{3}+A_{4}(1+\Delta \theta)^{\frac{3}{2}}-A_{5}(1+\Delta \theta)^{\frac{1}{2}}} \operatorname{Pr} .
\end{aligned}
$$

\section{Method of solution}

Spectral methods are a class of numerical methods used to solve differential equations arising in applied mathematics, science and engineering. The name spectral methods is derived from the fact that the solution is expressed as a 
series of orthogonal eigenfunctions of some linear operator. Although the SQLM has a very high convergence, it is limited to cases where there is only one independent variable. In this section, an efficient iterative spectral local linearization method (SLLM) which was proposed by Motsa [30] is used to numerically integrate the coupled nonlinear differential Eqs. (24) and (28) with the boundary condition Eqs. (26). To apply this technique, we consider the following nonlinear differential operators

$$
\begin{aligned}
\Omega_{f}= & \left(\exp \left(A_{1}+\alpha\left(1+\Delta \theta_{n}\right)\right)+\lambda\right) f_{n}^{\prime \prime \prime} \\
& +\alpha \Delta \exp \left(A_{1}+\alpha\left(1+\Delta \theta_{n}\right)\right) \theta_{n}^{\prime} f_{n}^{\prime \prime}-\lambda \delta\left(f_{n}^{\prime \prime}\right)^{2} f_{n}^{\prime \prime \prime} \\
& +A_{2}\left(\frac{2}{3} f_{n} f_{n}^{\prime \prime}-\frac{1}{3} f_{n}^{\prime 2}\right) \\
\Omega_{\theta}= & \left(A_{3}+A_{4}\left(1+\Delta \theta_{n}\right)^{\frac{3}{2}}-A_{5}\left(1+\Delta \theta_{n}\right)^{\frac{1}{2}}+\operatorname{Nr}\left(1+\Delta \theta_{n}\right)^{3}\right) \theta_{n}^{\prime \prime} \\
& +\Delta\left(\frac{3 A_{4}}{2}\left(1+\Delta \theta_{n}\right)^{\frac{1}{2}}-\frac{A_{5}}{2}\left(1+\Delta \theta_{n}\right)^{-\frac{1}{2}}+3 \operatorname{Nr}\left(1+\Delta \theta_{n}\right)^{2}\right) \theta_{n}^{\prime 2} \\
& +\frac{2}{3} \operatorname{Pr}_{v} f_{n} \theta_{n}^{\prime}\left(A_{3}+A_{4}\left(1+\Delta \theta_{n}\right)^{\frac{3}{2}}-A_{5}\left(1+\Delta \theta_{n}\right)^{\frac{1}{2}}\right) \\
& \exp \left(-A_{1}-\alpha\left(1+\Delta \theta_{n}\right)\right)=0 ;
\end{aligned}
$$

Eqs. (33) and (34) can be decoupled according to the following algorithm:

1. From Eq. (33) $\Omega_{f}$, solve $f_{n+1}$ assuming that $\theta_{n}$ is known from a previous iteration.

2. Solve $\theta_{n+1}$ from Eq. (34) $\Omega_{\theta}$ using the updated solution of $f_{n}$.

3. Subsequent iterative solutions are obtained by repeating steps 1 and 2.

In the framework of the SLLM, the following iterative scheme is obtained

$$
\begin{aligned}
& a_{1, n} f_{n+1}^{\prime \prime \prime}+a_{2, n} f_{n+1}^{\prime \prime}+a_{3, n} f_{n+1}^{\prime}+a_{4, n} f_{n+1}=R^{f}, \\
& a_{5, n} \theta_{n+1}^{\prime \prime}+a_{6, n} \theta_{n+1}^{\prime}+a_{7, n} \theta_{n+1}=R^{\theta}, \\
& f_{n+1}(0)=F_{w}, \quad f_{n+1}^{\prime}(0)=1, \quad f_{n+1}^{\prime}(\infty)=0, \\
& \theta_{n+1}(0)=1, \quad \theta_{n+1}(\infty)=0 .
\end{aligned}
$$

The coefficients in Eqs. (35) and (36) along with their righthand sides are defined as follows:

$$
\begin{aligned}
& a_{1, n}=\frac{\partial \Omega_{f}}{\partial f_{n}^{\prime \prime \prime}}=A_{1} \exp \left(A_{1}+\alpha\left(1+\Delta \theta_{n}\right)\right) \\
& +\lambda\left(1-\delta f_{n}^{\prime \prime 2}\right), \quad \alpha_{3, n}=\frac{\partial \Omega_{f}}{\partial f_{n}^{\prime}} \\
& =-2 / 3 A_{2} f_{n^{\prime}}^{\prime} \\
& a_{2, n}=\frac{\partial \Omega_{f}}{\partial f_{n}^{\prime \prime}}=\alpha \Delta \exp \left(A_{1}+\alpha\left(1+\Delta \theta_{n}\right)\right) \theta_{n}^{\prime}-2 \delta \lambda f_{n}^{\prime \prime} f_{n}^{\prime \prime \prime} \\
& +\frac{2}{3} A_{2} f_{n}, \quad a_{4, n}=\frac{\partial \Omega_{f}}{\partial f_{n}}=\frac{2}{3} A_{2} f_{n}^{\prime \prime}, \\
& \alpha_{5, n}=\frac{\partial \Omega_{\theta}}{\partial \theta_{n}^{\prime \prime}}=A_{3}+A_{4}\left(\Delta \theta_{n}+1\right)^{\frac{3}{2}}-A_{5}\left(\Delta \theta_{n}+1\right)^{\frac{1}{2}} \\
& +\operatorname{Nr}\left(\Delta \theta_{n}+1\right)^{3} \\
& \alpha_{6, n}=\frac{\partial \Omega_{\theta}}{\partial \theta_{n}^{\prime}},=2 \Delta\left(\frac{3}{2} A_{4}\left(\Delta \theta_{n}+1\right)^{\frac{1}{2}}-\frac{1}{2} A_{5}\left(\Delta \theta_{n}+1\right)^{-\frac{1}{2}}\right. \\
& \left.+3 \operatorname{Nr}\left(\Delta \theta_{n}+1\right)^{2}\right) \theta_{n}^{\prime}+\frac{2}{3} \operatorname{Pr}_{v} f_{n}\left(A_{3}+A_{4}\left(\Delta \theta_{n}+1\right)^{\frac{3}{2}}\right. \\
& \left.-A_{5}\left(\Delta \theta_{n}+1\right)^{\frac{1}{2}}\right) \exp \left(-A_{1}-\alpha\left(1+\Delta \theta_{n}\right)\right) \\
& \alpha_{7, n}=\frac{\partial \Omega_{\theta}}{\partial \theta_{n}}=\Delta\left(\frac{3}{2} A_{4}\left(1+\Delta \theta_{n}\right)^{\frac{1}{2}}-\frac{1}{2} A_{5}\left(\Delta \theta_{n}+1\right)^{-\frac{1}{2}}\right. \\
& \left.+3 \operatorname{Nr}\left(\Delta \theta_{n}+1\right)^{2}\right) \theta_{n}^{\prime \prime}+\Delta^{2}\left(\frac{3}{4} A_{4}\left(1+\Delta \theta_{n}\right)^{-\frac{1}{2}}\right. \\
& \left.+\frac{1}{4} A_{5}\left(\Delta \theta_{n}+1\right)^{-\frac{3}{2}}+6 \mathrm{Nr}\left(\Delta \theta_{n}+1\right)\right) \theta_{n}^{\prime 2} \\
& +\frac{1}{3} \operatorname{Pr}_{v} f_{n} \theta_{n}^{\prime} \Delta \exp \left(-A_{1}-\alpha\left(1+\Delta \theta_{n}\right)\right)\left[3 A_{4}\left(\Delta \theta_{n}+1\right)^{\frac{1}{2}}\right. \\
& -A_{5}\left(\Delta \theta_{n}+1\right)^{-\frac{1}{2}} \\
& \left.-2 \alpha\left(A_{3}+A_{4}\left(\Delta \theta_{n}+1\right)^{\frac{3}{2}}-A_{5}\left(\Delta \theta_{n}+1\right)^{\frac{1}{2}}\right)\right], \\
& R^{f}=a_{1, n} f_{n}^{\prime \prime \prime}+a_{2, n} f_{n}^{\prime \prime}+a_{3, n} f_{n}^{\prime}+a_{4, n} f_{n}-\Omega_{f}, \\
& R^{\theta}=a_{5, n} \theta_{n}^{\prime \prime}+a_{6, n} \theta_{n}^{\prime}+a_{7, n} \theta_{n}-\Omega_{\theta} \text {. }
\end{aligned}
$$

Equations (35)-(37) are integrated numerically using the Chebyshev pseudo-spectral technique (see Mondal et al. [31], Motsa et al. [32] and Maleki et al. [33]). In order to apply this technique, the semi-infinite domain $\eta \in[0, \infty)$ is replaced with a truncated domain $\eta \in\left[0, \varpi_{\infty}\right]$, where $\varpi_{\infty} \in \mathbb{Z}^{+}$is a mapping parameter. Using the transformation $\eta=\frac{1}{2}(\xi+1) \varpi_{\infty}$, the interval $\left[0, \varpi_{\infty}\right]$ is mapped to $[-1,1]$ which the Chebyshev pseudo-spectral technique can be used. The unknown functions $f(\eta)$ and $\theta(\eta)$ are 
discretized using the Chebyshev-Gauss-Lobatto collocation points:

$\xi_{k}=\cos \left(\frac{\pi k}{\bar{N}}\right), \quad k=0,1, \ldots, \bar{N} ; \quad-1 \leq \xi \leq 1$.

The derivatives of $f(\eta)$ and $\theta(\eta)$ are computed using the Chebyshev differentiation matrix $D$, at the collocation points as a matrix vector product, that is:

$\frac{\mathrm{d} f}{\mathrm{~d} \eta}=\sum_{i=0}^{\bar{N}} D_{i j} f\left(\xi_{i}\right)=\mathbf{D} F, \quad j=0,1,2, \ldots, \bar{N}$,

where $\bar{N}+1$ is the number of collocation points, $\mathbf{D}=2 D / \varpi_{\infty}$ and $F=\left[f\left(\xi_{0}\right), f\left(\xi_{1}\right), \ldots, f\left(\xi_{\bar{N}}\right)\right]^{T}$ is a vector function at the collocation point.

Let $\Theta$ be a similarity vector function representing $\theta$. The higher-order derivatives of $f$ and $\theta$ are evaluated as powers of $\mathbf{D}$, that is

$f^{s}(\eta)=\mathbf{D}^{s} F, \quad \theta^{s}(\eta)=\mathbf{D}^{s} \Theta$.

Substituting Eqs. (39)-(41) into Eqs. (35)-(37) yields the following decoupled matrices

$$
\begin{aligned}
& {\left[\begin{array}{ccc}
\mathbf{D}_{1,1} & \ldots & \mathbf{D}_{1, \bar{N}+1} \\
\operatorname{diag}\left[a_{1, n}\right] \mathbf{D}^{3}+\operatorname{diag}\left[a_{2, n}\right] \mathbf{D}^{2}+\operatorname{diag}\left[a_{3, n}\right] \mathbf{D}+\operatorname{diag}\left[a_{4, n}\right] \mathbf{I} \\
\mathbf{D}_{\bar{N}, 1} & \cdots & \mathbf{D}_{\bar{N}, \bar{N}+1} \\
0 & \cdots & 1
\end{array}\right]} \\
& \times\left[\begin{array}{c}
f_{n+1}\left(\xi_{0}\right) \\
f_{n+1}\left(\xi_{1}\right) \\
\vdots \\
f_{n+1}\left(\xi_{\bar{N}-1}\right) \\
f_{n+1}\left(\xi_{\bar{N}}\right)
\end{array}\right]=\left[\begin{array}{c}
0 \\
R_{n+1}^{f}\left(\xi_{1}\right) \\
\vdots \\
1 \\
F_{w}
\end{array}\right] \\
& {\left[\begin{array}{ccc}
1 & 0 \ldots & 0 \\
\operatorname{diag}\left[a_{5, n}\right] \mathbf{D}^{2}+\operatorname{diag}\left[a_{6, n}\right] \mathbf{D}+\operatorname{diag}\left[a_{7, n}\right] \mathbf{I} \\
0 & \ldots & 1
\end{array}\right]\left[\begin{array}{c}
\theta_{n+1}\left(\xi_{0}\right) \\
\vdots \\
\theta_{n+1}\left(\xi_{\bar{N}}\right)
\end{array}\right]} \\
& =\left[\begin{array}{c}
0 \\
R_{n+1}^{\theta}\left(\xi_{1}\right) \\
\vdots \\
1
\end{array}\right]
\end{aligned}
$$

Here $\mathbf{I}$ is an $(\bar{N}+1) \times(\bar{N}+1)$ identity matrix, and diag[] denotes a diagonal matrix. A suitable initial approximation for the SLLM scheme is

$f_{0}(\eta)=F_{w}+1-\exp (-\eta), \quad \theta_{0}(\eta)=\exp (-\eta)$,

\section{Numerical validation}

The cases $\lambda=A_{1}=A_{4}=A_{5}=0, \theta_{w}=A_{2}=1$ and $A_{3}=1$ correspond to a Newtonian fluid with constant viscosity, thermal conductivity and linear thermal radiation which has been studied by Jalil and Asghar [16] using the Kellerbox method. To validate the correctness of the numerical results obtained from the iterative scheme given by Eqs. (35-37), the skin friction coefficient $f^{\prime \prime}(0)$ at the seventh iterates with $N=80$ is compared with Jalil and Asghar [16] in Table 3. It is apparent that there is a good agreement between the two results.

\section{Results and discussion}

In this section, we give the results of the numerical simulation and the effects of the nanoparticle volume fraction, $\phi$, fluid parameter, $\lambda$, suction/injection parameter, $F_{w}$, thermal radiation parameter, $\mathrm{Nr}$, and temperature ratio parameter, $\theta_{w}$ on the nanofluid velocity profile, $f^{\prime}(\eta)$, temperature profile, $\theta(\eta)$, skin friction coefficient and Nusselt number are performed and discussed. The range of parameters is as follows: $1 \% \leq \phi \leq 10 \%, 0 \leq \lambda \leq 5,0 \leq \delta \leq 3,1.2 \leq \theta_{w} \leq 2.1$ and $-1 \leq F_{w} \leq 1$. (See Jalil and Asghar [16] and Sandeep and Gnaneswara [7]). The default parameter values used in simulating the velocity and temperature profiles are $\lambda=\delta=\mathrm{Nr}=F w=1, \phi=0.1, \theta_{w}=1.5$ and $\operatorname{Pr}_{v}=6.96$. Therefore, where these parameter values are not explicitly stated, it will be understood that such a parameter is assigned the default value.

The nanoparticle volume fraction quantifies the amount of the $\mathrm{Al}_{2} \mathrm{O}_{3}$ nanoparticles contained in the synthesized nanofluid. The nanofluid velocity and temperature profiles for different values of nanoparticle volume fraction are displayed in Fig. 1. We observed that both the velocity and temperature profiles are enhanced with an increase in the value of nanoparticle volume fraction. Also, the

\begin{tabular}{|c|c|c|c|}
\hline \multirow[t]{2}{*}{$F_{w}$} & \multicolumn{3}{|l|}{$f^{\prime \prime}(0)$} \\
\hline & Jalil and Asghar [16] & SLLM & $\begin{array}{l}\text { Relative } \\
\text { error }\end{array}$ \\
\hline 0.75 & 0.984436 & 0.98443940 & 0 \\
\hline 0.50 & 0.873640 & 0.87364290 & 0 \\
\hline 0 & 0.677647 & 0.67764824 & 0 \\
\hline-0.50 & 0.518869 & 0.51887049 & 0 \\
\hline-0.75 & 0.453523 & 0.45352500 & 0 \\
\hline
\end{tabular}

Table 3 Comparison of the SLLM results for $-f^{\prime \prime}(0)$ with Jalil and Asghar [16] for distinct values of $F_{w}$ when $\lambda=A_{1}=A_{4}=A_{5}=0$, $\theta_{w}=A_{2}=1$ and $A_{3}=1$ 


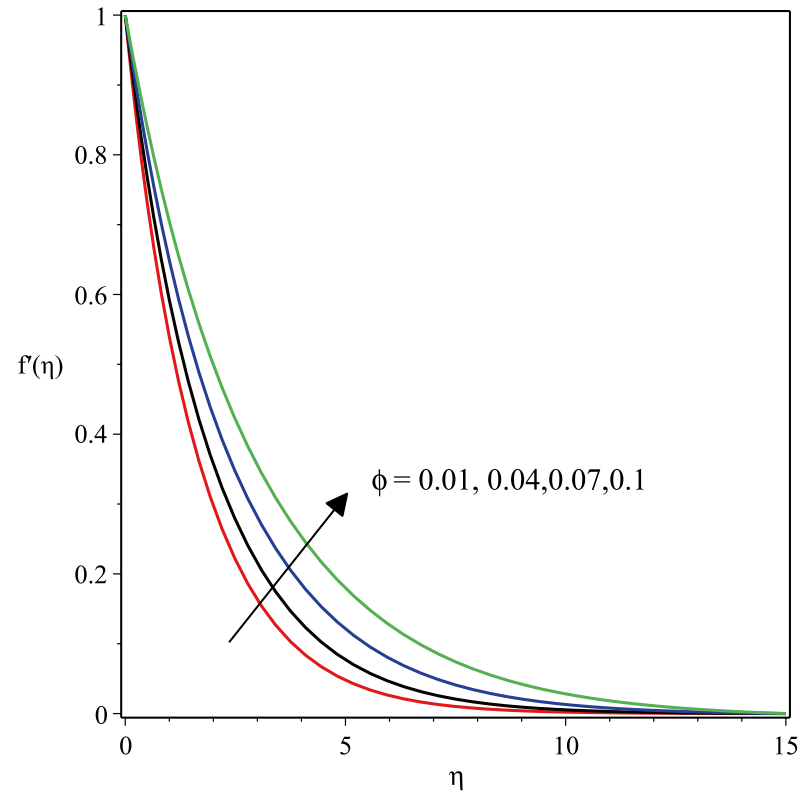

(a)

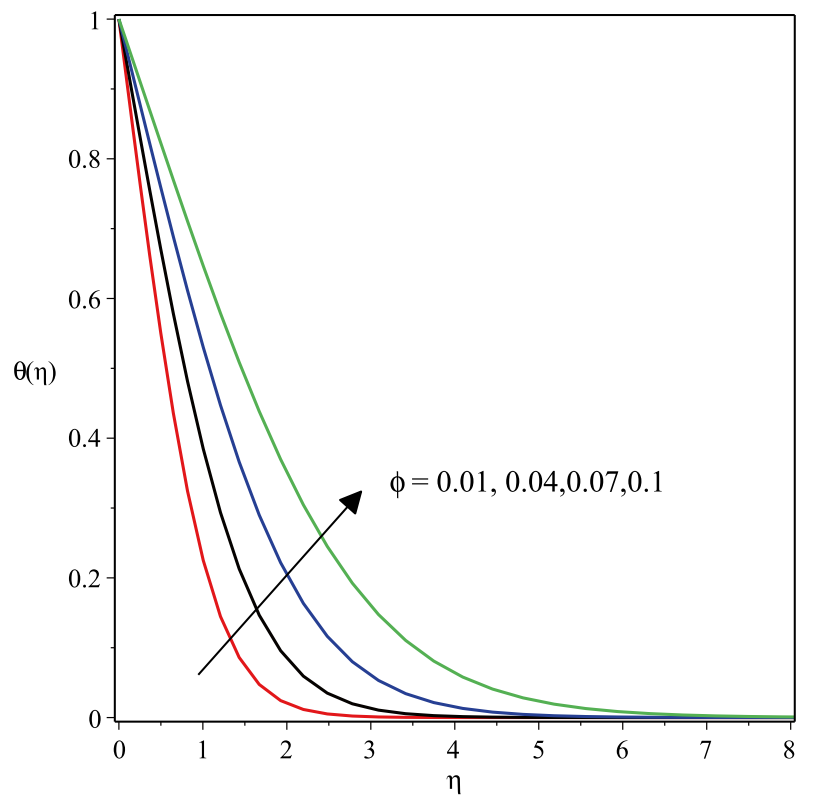

(b)

Fig. 1 Effect of the nanoparticle volume fraction on the velocity and temperature profiles

momentum and thermal boundary layers become thicker with an increase in the nanoparticle volume fraction. Figure 2 shows the effect of the fluid parameter on the nanofluid velocity profiles. It is seen that with an increase in the fluid parameter, the nanofluid velocity profiles and

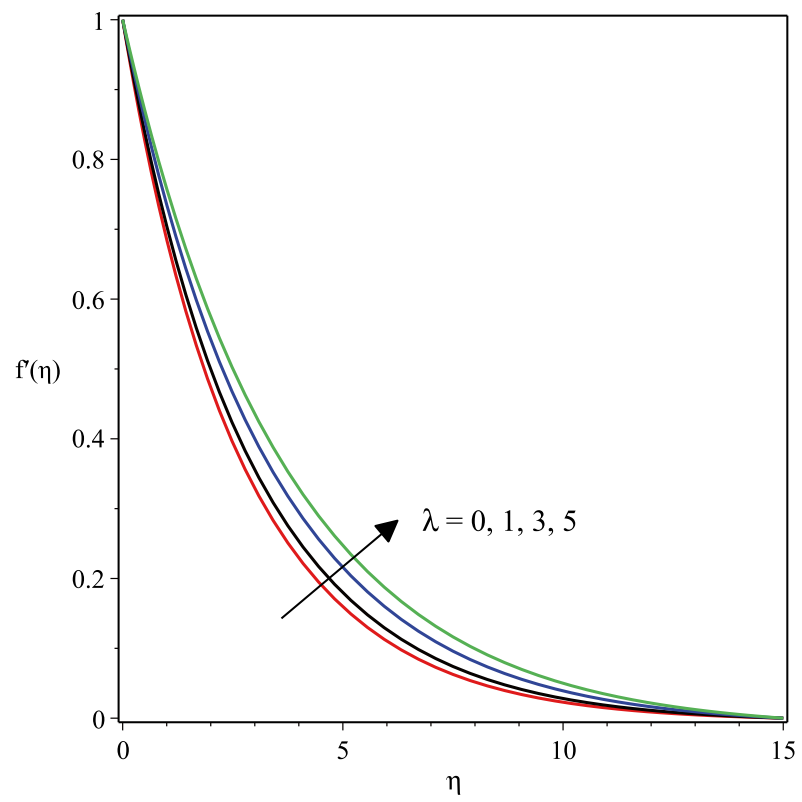

Fig. 2 Effect of the fluid parameter on the velocity profile

\section{SN Applied Sciences}

the momentum boundary layer thickness are enhanced. Physically, this is correct since the fluid parameter has an inverse relation with the nanofluid dynamic viscosity; thus, the fluid becomes less viscous with large value of the fluid parameter. Hence, the velocity profiles are enhanced. This

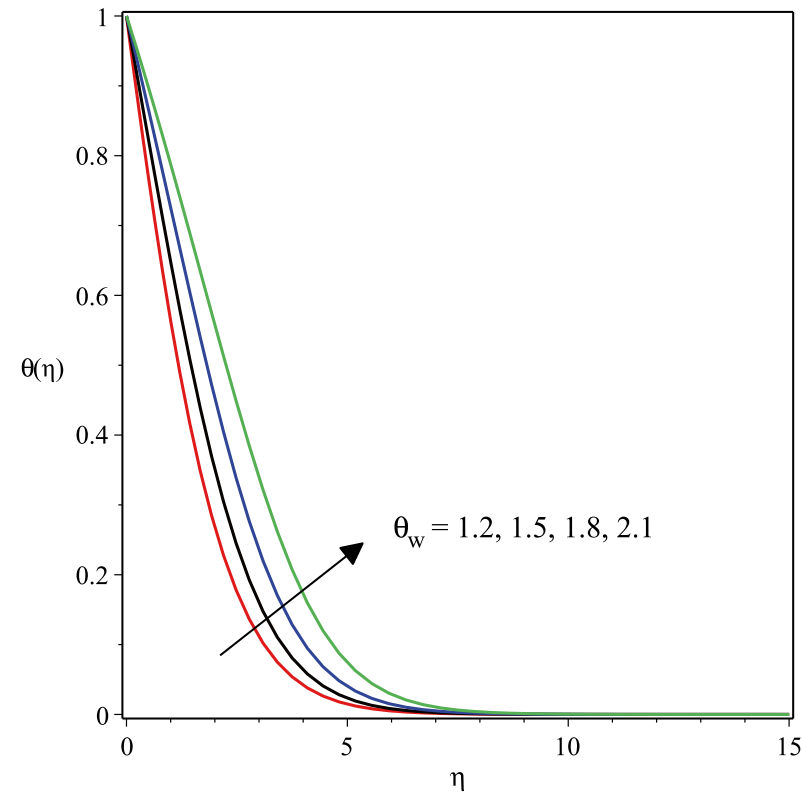

Fig. 3 Effect of the nanoparticle volume fraction on the temperature profile 


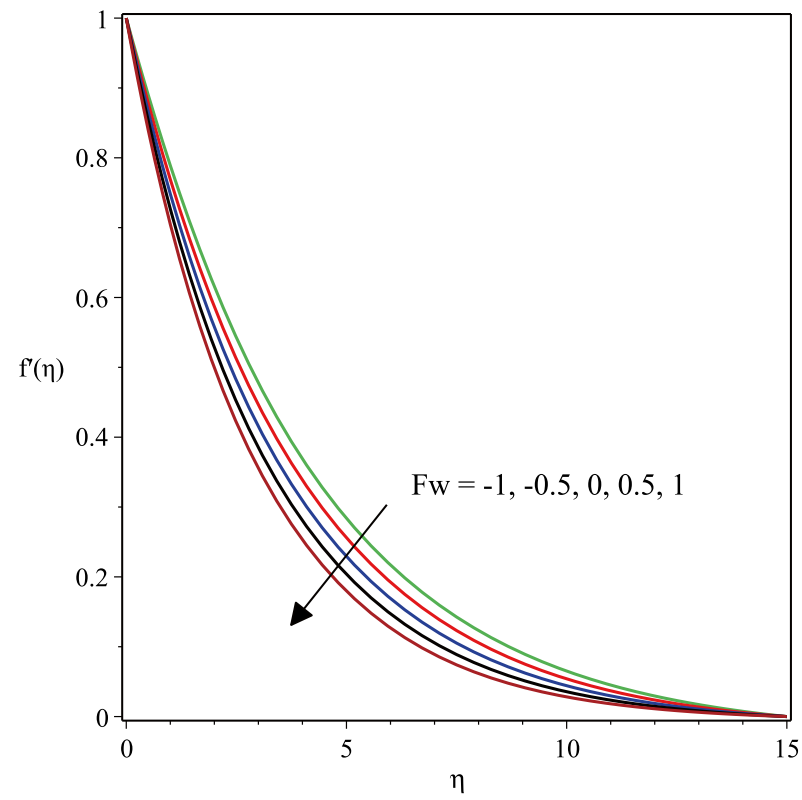

(a)

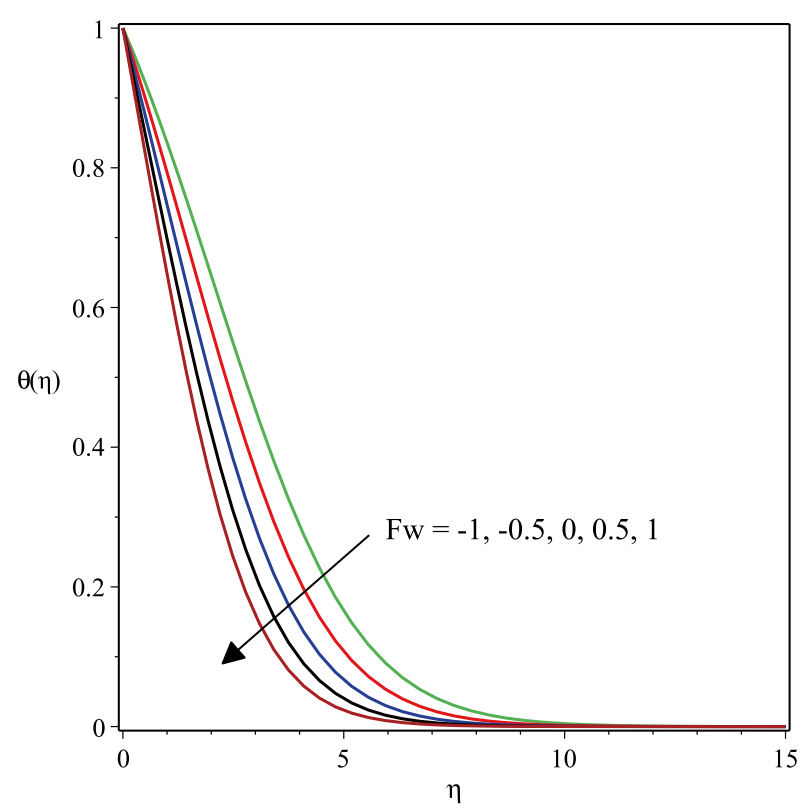

(b)

Fig. 4 Effect of the suction/injection parameter on the velocity and temperature profile

finding is consistent with Javed et al. [23] for the case of a pure fluid.

Figure 3 shows the effect of the temperature ratio parameter on the nanofluid temperature profiles. We observed that the temperature profiles, as well as the

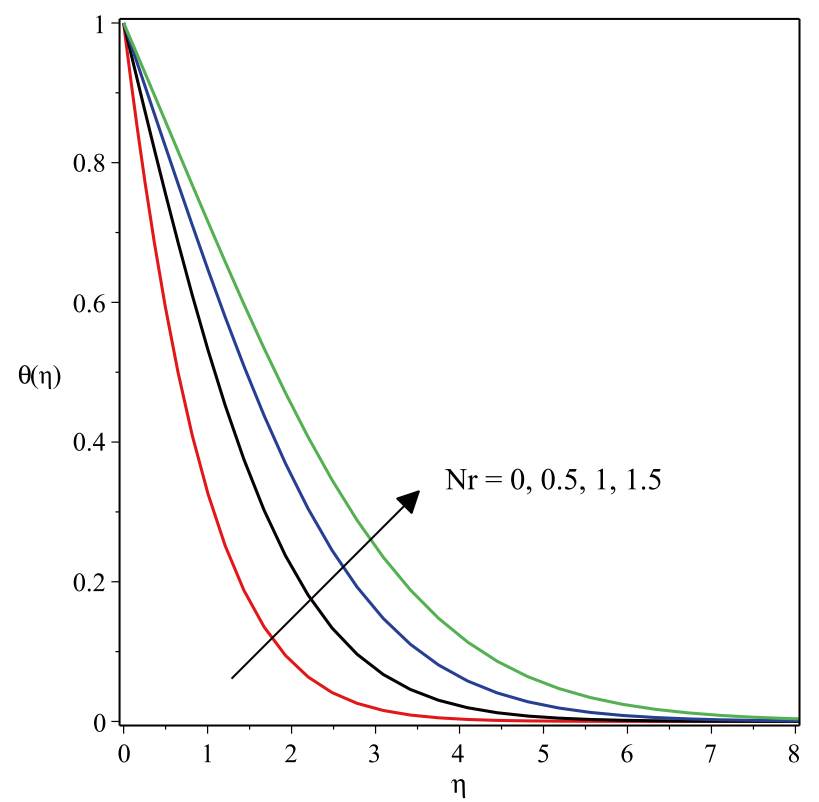

Fig. 5 Effect of the thermal radiation parameter on the temperature profile thermal boundary layer thickness, are enhanced with an increase in the value of the temperature ratio parameter. This trend is in agreement with Sandeep and Gnaneswara [7].

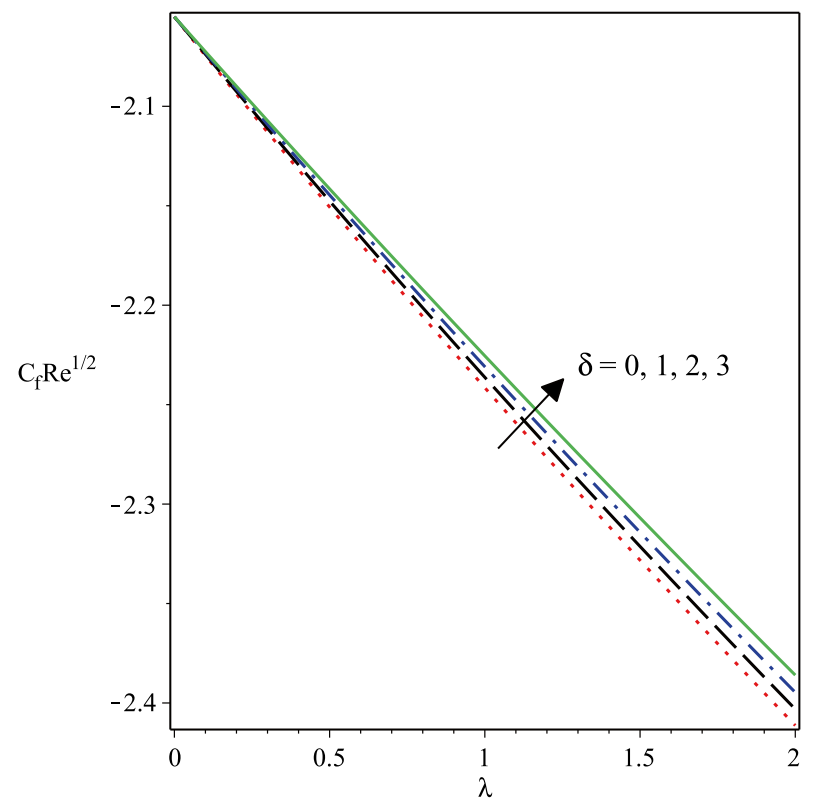

Fig. 6 Effect of the fluid parameter, $\lambda$, on the skin friction coefficient for different values of the fluid parameter, $\delta$ 


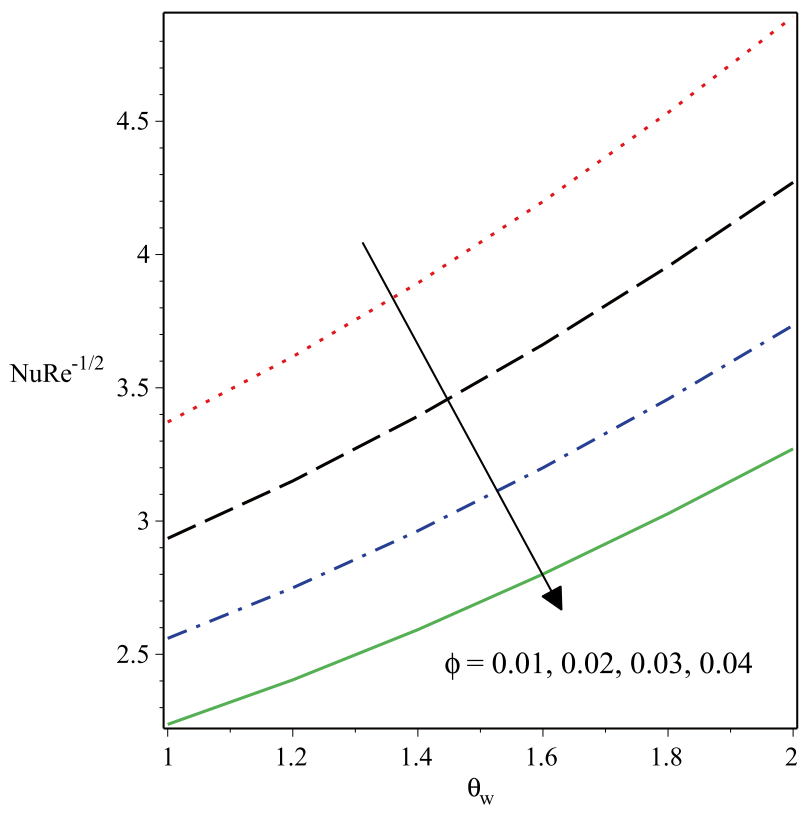

Fig. 7 Effect of the temperature ratio parameter on the Nusselt number for different values of nanoparticle volume fraction

Figure 4 shows the effect of the suction $\left(f_{w}>0\right)$ and injection $\left(f_{w}<0\right)$ parameter on the nanofluid velocity and temperature profiles. From Fig. $4 \mathrm{a}$, it is seen that the

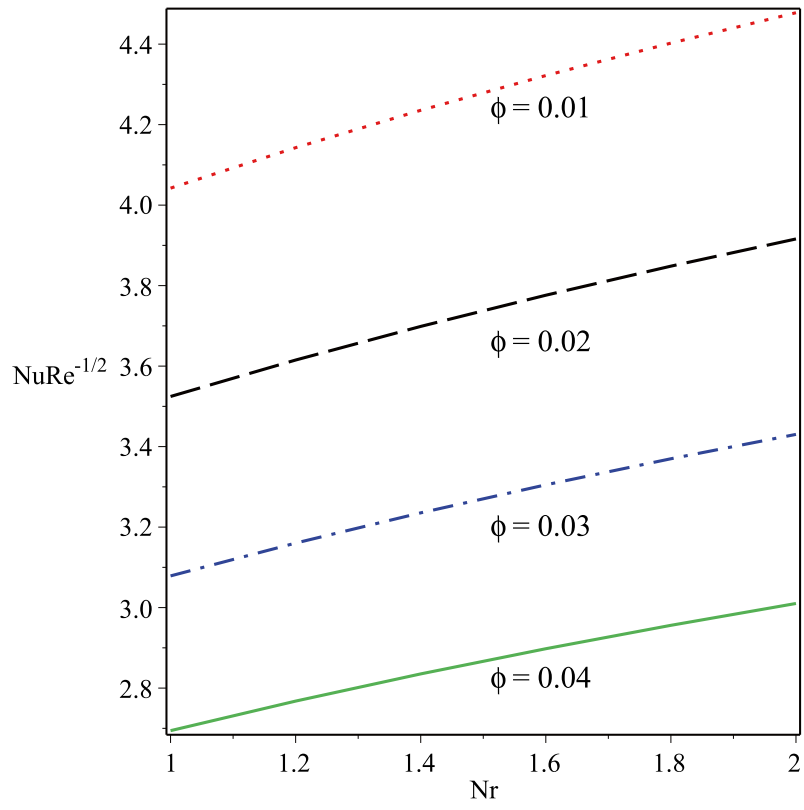

Fig. 8 Effect of the thermal radiation parameter on the Nusselt number for different values of nanoparticle volume fraction

velocity profiles, as well as the thickness of the momentum boundary layer, decrease with an increase in the suction parameter. However, the velocity and momentum
Table 4 The computed results for skin friction coefficient, $C_{f}$, and Nusselt number, $\mathrm{Nu}$, for $\mathrm{Al}_{2} \mathrm{O}_{3}$ /water nanofluid for varying the parameters: $\phi, \lambda, \delta, f w, \theta_{w}$ and $\mathrm{Nr}$

\begin{tabular}{|c|c|c|c|c|c|c|c|}
\hline$\phi$ & $\lambda$ & $\delta$ & $f w$ & $\theta_{w}$ & $\mathrm{Nr}$ & $C_{f}$ & $\mathrm{Nu}$ \\
\hline 0.01 & 1 & 1 & 1 & 1.5 & 1 & -1.31566819 & 4.04218773 \\
\hline 0.04 & & & & & & -1.53640389 & 2.69425801 \\
\hline 0.07 & & & & & & -1.83309969 & 1.82571968 \\
\hline \multirow[t]{21}{*}{0.1} & & & & & & -2.23626267 & 1.25732143 \\
\hline & 0 & & & & & -2.05499683 & 1.24567815 \\
\hline & 1 & & & & & -2.23626267 & 1.25732143 \\
\hline & 3 & & & & & -2.55833356 & 1.27519931 \\
\hline & 5 & & & & & -2.84185954 & 1.28832024 \\
\hline & & 1 & & & & -2.23626267 & 1.25732143 \\
\hline & & 1.5 & & & & -2.23360279 & 1.25715057 \\
\hline & & 2 & & & & -2.23091741 & 1.25697829 \\
\hline & & 2.5 & & & & -2.22820586 & 1.25680457 \\
\hline & & & -1 & & & -1.47135446 & 0.51499994 \\
\hline & & & -0.5 & & & -1.64056501 & 0.67145981 \\
\hline & & & 0.5 & & & -2.02355151 & 1.04510263 \\
\hline & & & 1 & & & -2.23626267 & 1.25732143 \\
\hline & & & & 1.2 & & -2.03895232 & 1.11810439 \\
\hline & & & & 1.5 & & -2.23626267 & 1.25732143 \\
\hline & & & & 1.8 & & -2.43248202 & 1.41661565 \\
\hline & & & & 2.1 & & -2.62786066 & 1.59104731 \\
\hline & & & & & 0 & -2.27747031 & 0.97952469 \\
\hline & & & & & 0.5 & -2.25163761 & 1.14019626 \\
\hline & & & & & 1 & -2.23626267 & 1.25732143 \\
\hline & & & & & 1.5 & -2.22583993 & 1.34955641 \\
\hline
\end{tabular}


boundary layers are enhanced with an increase in the injection parameter. Similarly, the temperature profiles and the thermal boundary layer thickness reduce with an increase in the suction parameter, whereas an opposite trend is observed for the case of injection as shown in Fig. 4b. This result is in agreement with the findings of Jalil and Asghar [16].

The impact of the thermal radiation parameter on the nanofluid temperature profiles is presented in Fig. 5. From this plot, the nanofluid temperature profile is an increasing function of the thermal radiation parameter. The physical reason for this observed trend is that, for a higher value of the radiation parameter, more heat is transferred to the nanofluid since the mean absorption coefficient $k_{m}$ reduces with an increase in the radiation parameter. This temperature profile is similar to Ramzan et al. [27] for the case of pure fluid.

Figure 6 displays the effect of the fluid parameter, $\lambda$, on the skin friction for distinct values of the fluid parameter, $\delta$. Clearly, it is seen that the skin friction coefficient increases with an increase $\delta$. The influence of the temperature ratio parameter and the thermal radiation parameter on the Nusselt number is displayed in Figs. 7 and 8 . It is evident from these plots that the Nusselt number increases with an increase in the values of $\theta_{w}$ and $\mathrm{Nr}$ (Table 4).

\section{Conclusions}

The flow and heat transfer in a Powell-Eyring nanofluid flow past a stretching surface have been studied. The similarity solution to the model describing the nanofluid flow and energy balance was found using the Lie group analysis. An iterative spectral local linearization method was used to solve the conservation equations. The effects of nanoparticles, thermal radiation and suction/injection have been considered in the problem. The effects of these parameters on the nanofluid velocity and temperature profiles, as well as the skin friction coefficient and Nusselt number, are determined and discussed. A summary of the results of the study is as follows:

1. The velocity profiles are enhanced with an increase in the fluid parameter, $\lambda$ and nanoparticle volume fraction.

2. The velocity and temperature profiles are decreasing functions of the suction parameter, while injection shows an opposite trend.

3. An increase in the nanoparticle volume fraction, thermal radiation parameter and temperature ratio parameter enhances the thermal boundary layer thickness as well as the temperature profiles.
4. The skin friction coefficient increases with an increase in the fluid parameter, $\delta$.

5. Increasing the temperature ratio parameter and thermal radiation parameter increases the Nusselt number.

Funding The authors are grateful to the University of KwaZulu-Natal, South Africa, for financial support.

\section{Compliance with ethical standards}

Conflict of interest The authors declare that they have no conflict of interest.

\section{References}

1. Wong KV, De Leon O (2010) Applications of nanofluids: current and future. Adv Mech Eng 2:519659

2. Robert T, Sylvain C, Todd O, Patrick P, Andrey G, Wei L, Gary R, Ravi P, Himanshu T (2013) Small particles, big impacts: a review of the diverse applications of nanofluids. J Appl Phys 113(1):011301

3. Devendiran DK, Amirtham VA (2016) A review on preparation, characterization, properties and applications of nanofluids. Renew Sustain Energy Rev 60:21-40 ISSN 1364-0321

4. Munyalo JM, Zhang X (2018) Particle size effect on thermophysical properties of nanofluid and nanofluid based phase change materials: a review. J Mol Liq 265:77-87

5. Khan WA, Pop I (2010) Boundary-layer flow of a nanofluid past a stretching sheet. Int J Heat Mass Transf 53:2477-2483

6. Turkyilmazoglu M (2012) Exact analytical solutions for heat and mass transfer of MHD slip flow in nanofluids. Chem Eng Sci 84:182-187

7. Sandeep N, Gnaneswara RM (2017) Heat transfer of nonlinear radiative magnetohydrodynamic cu-water nanofluid flow over two different geometries. J Mol Liq 225:87-94

8. Dhlamini M, Mondal H, Sibanda P, Motsa S (2018) Spectral quasilinearization methods for Powell-Eyring mhd flow over a nonlinear stretching surface. J Nanofluids 7(5):917-927

9. Das S, Mondal H, Kundu PK, Sibanda P (2019) Spectral quasilinearization method for Casson fluid with homogeneous heterogeneous reaction in presence of nonlinear thermal radiation over an exponential stretching sheet. Multidiscip Model Mater Struct 15(2):398-417

10. Afify A A, El-Aziz M Abd (2017) Lie group analysis of flow and heat transfer of non-Newtonian nanofluid over a stretching surface with convective boundary condition. Pramana 88(2):31

11. Das K, Acharya N, Kundu PK (2018) Influence of variable fluid properties on nanofluid flow over a wedge with surface slip. Arab J Sci Eng 43(5):2119-2131. https://doi.org/10.1007/s1336 9-017-2499-x

12. Ogunseye HA, Sibanda P, Mondal H (2019) Mhd mixed convective stagnation-point flow of Eyring-Powell nanofluid over stretching cylinder with thermal slip conditions. J Central South Univ 26(5):1172-1183

13. Masoud HS, Moghadassi AR, Henneke DE (2010) A new dimensionless group model for determining the viscosity of nanofluids. J Therm Anal Calorim 100(3):873-877. https://doi. org/10.1007/s10973-010-0721-0 
14. Hassani S, Saidur R, Mekhilef S, Hepbasli A (2015) A new correlation for predicting the thermal conductivity of nanofluids; using dimensional analysis. Int J Heat Mass Transf 90:121-130. https:// doi.org/10.1016/j.ijheatmasstransfer.2015.06.040

15. Akgül MB, Pakdemirli MM (2012) Lie group analysis of a non-newtonian fluid flow over a porous surface. Sci Iran 19(6):1534-1540

16. Jalil M, Asghar S (2013) Flow and heat transfer of Powell-Eyring fluid over a stretching surface: a lie group analysis. J Fluids Eng 135(12):121201-121206

17. Rehman K, Saba N, Malik MY, Malik AA (2017) Encountering heat and mass transfer mechanisms simultaneously in PowellEyring fluid through lie symmetry approach. Case Stud Therm Eng 10:541-549

18. Pakdemirli M, Yurusoy M (1998) Similarity transformations for partial differential equations. SIAM Rev 40(1):96-101

19. Hayat $T$, Herman Mambili-Mamboundou $H$, Momoniat $E$, Mahomed F M (2008) The Rayleigh problem for a third grade electrically conducting fluid in a magnetic field. J Nonlinear Math Phys 15(sup 1):77-90

20. Abd-el Malek MB, Hassan HS (2010) Symmetry analysis for steady boundary-layer stagnation-point flow of Rivlin-Ericksen fluid of second grade subject to suction. Nonlinear Anal: Modell Control 15(4):379-396

21. Roşca NC, Roşca AV, Pop I (2016) Lie group symmetry method for mhd double-diffusive convection from a permeable vertical stretching/shrinking sheet. Comput Math Appl 71(8):1679-1693

22. Powell RE, Eyring $\mathrm{H}$ (1944) Mechanisms for the relaxation theory of viscosity. Nature 154(1):427-428

23. Javed T, Ali N, Abbas Z, Sajid M (2013) Flow of an Eyring-Powell non-Newtonian fluid over a stretching sheet. Chem Eng Commun 200(3):327-336

24. Hayat T, Iqbal Z, Qasim M, Obaidat S (2012) Steady flow of an Eyring-Powell fluid over a moving surface with convective boundary conditions. Int J Heat Mass Transf 55(7):1817-1822

25. Mahanthesh B, Gireesha BJ, Gorla RSR (2017) Unsteady threedimensional MHD flow of a nano Eyring-Powell fluid past a convectively heated stretching sheet in the presence of thermal radiation, viscous dissipation and Joule heating. J Assoc Arab Univ Basic Appl Sci 23:75-84

26. Agbaje TM, Mondal S, Motsa SS, Sibanda P (2017) A numerical study of unsteady non-Newtonian Powell-Eyring nanofluid flow over a shrinking sheet with heat generation and thermal radiation. Alex Eng J 56(1):81-91

27. Ramzan M, Bilal M, Kanwal S, Chung JD (2017) Effects of variable thermal conductivity and non-linear thermal radiation past an Eyring-Powell nanofluid flow with chemical reaction. Commun Theor Phys 67(6):723

28. Khanafer K, Vafai K (2011) A critical synthesis of thermophysical characteristics of nanofluids. Int J Heat Mass Transf 54(19):4410-4428

29. Pantokratoras A (2004) Further results on the variable viscosity on flow and heat transfer to a continuous moving flat plate. Int J Eng Sci 42(17):1891-1896

30. Motsa SS (2013) A new spectral local linearization method for nonlinear boundary layer flow problems. J Appl Math Article ID 423628, 15 pages

31. Mondal H, Almakki M, Sibanda P (2019) Dual solutions for three-dimensional magnetohydrodynamic nanofluid flow with entropy generation. J Comput Des Eng 6(4):657-665. https:// doi.org/10.1016/j.jcde.2019.01.003

32. Motsa SS, Sibanda P, Shateyi S (2011) On a new quasi-linearization method for systems of nonlinear boundary value problems. Math Methods Appl Sci 34(11):1406-1413. https://doi. org/10.1002/mma.1449

33. Maleki M, Hashim I, Abbasbandy S (2012) Analysis of IVPS and BVPS on semi-infinite domains via collocation methods. J Appl Math Article ID 696574, 21 pages

Publisher's Note Springer Nature remains neutral with regard to jurisdictional claims in published maps and institutional affiliations. 